\title{
REAÇÃO DE CLONES DE UMEZEIRO (Prunus mume Sieb. et Zucc.) E CULTIVARES DE PESSEGUEIRO A Meloidogyne javanica (Treub, 1885) Chitwood, 1949'
}

\author{
NEWTON ALEX MAYER ${ }^{2}$, FERNANDO MENDES PEREIRA ${ }^{3}$, JAIME MAIA DOS SANTOS ${ }^{4}$
}

\begin{abstract}
RESUMO - Um amplo projeto de estudos sobre a utilização do umezeiro como porta-enxerto para pessegueiro está sendo desenvolvido na FCAV/ UNESP, Câmpus de Jaboticabal-SP, devido, especialmente, às promissoras características para uso como redutor de vigor da copa e sua boa qualidade de frutos. Alguns trabalhos na literatura citam o umezeiro como resistente ao nematóide das galhas, entretanto dispõe- se de poucas informações. Neste trabalho, teve-se por objetivo estudar a reação de clones de umezeiro e cultivares de pessegueiro a Meloidogyne javanica. O experimento foi conduzido em casa de vegetação, com 6 tratamentos (Clones 05; 10 e 15 de umezeiro e as cultivares Okinawa, Aurora-1 e Dourado-1 de pessegueiro) e 9 repetições. As plantas foram mantidas em vasos de cerâmica contendo uma mistura de solo e areia $(1: 1, \mathrm{v} / \mathrm{v})$, previamente autoclavada a $121^{\circ} \mathrm{C}$ e $1 \mathrm{kgf.cm}{ }^{-2}$ por 2 horas. Aos sessenta dias após o plantio, cada planta foi inoculada com 3.000 ovos e juvenis de segundo estádio de Meloidogyne javanica. Aos 100 dias após a inoculação, as plantas foram colhidas para avaliação da massa de matéria fresca do sistema radicular, número de galhas por sistema radicular, número de ovos e juvenis por $10 \mathrm{~g}$ de raízes, número de ovos e juvenis por sistema radicular e fator de reprodução. Verificou-se que todos os clones e cultivares de umezeiro e pessegueiro, respectivamente, mostraram-se resistentes a Meloidogyne javanica.
\end{abstract}

Termos para indexação: Porta-enxerto, frutas de caroço, nematóide de galhas, Prunus spp., Meloidogyne spp.

\section{REACTION OF JAPANESE APRICOT CLONES (Prunus mume Sieb. et Zucc.) AND PEACH TREE CULTIVARS TO} Meloidogyne javanica (Treub, 1885) Chitwood, 1949

\begin{abstract}
A wide science project on the use of the japanese apricot as rootstock for peach tree is being developed at Faculdade de Ciências Agrárias e Veterinárias - UNESP, Jaboticabal Campus, São Paulo State, Brazil, due, especially, to the promising characteristics for using as canopy vigor reducer and its good fruit quality. Some works in the literature mention the japanese apricot as resistant to the root-knot nematodes, however the information are still scanty. The objective of this work was to study the reaction of japanese apricot clones and peach tree cultivars to Meloidogyne javanica. The experiment was carried out under greenhouse conditions. There were 6 treatments (the japanese apricot clones 05,10 and 15 ; and Okinawa, Aurora-1 and Dourado-1 peach tree cultivars) and 9 replications. The plants were maintained in ceramic boxes containing a soil-sand mixture $(1: 1, \mathrm{v} / \mathrm{v})$, previously autoclaved at $121^{\circ} \mathrm{C}$ and $1 \mathrm{kgf} . \mathrm{cm}^{-2}$ for 2 hours. On the sixtyth day after planting, each plant was inoculated with 3.000 eggs and second stage juvenile of Meloidogyne javanica. A hundred days after inoculation the plants were harvested for evaluation of root fresh matter mass, root gall, egg and juvenile number in a $10 \mathrm{~g}$ sample, egg and juvenile number for the whole radicular system and reproduction factor (RF). It was verified that all the japanese apricot clones and peach tree cultivars were resistant to Meloidogyne javanica.
\end{abstract}

Index terms: Rootstock, stone fruits, root-knot nematode, Prunus spp., Meloidogyne spp.

No Brasil, a cultura do pessegueiro ocupa uma área de 22.039 ha, distribuída, principalmente, nos Estados do Rio Grande do Sul (14.344 ha), Santa Catarina (3.056 ha), São Paulo (2.133 ha), Paraná (1.796 ha) e Minas Gerais (696 ha). A produção brasileira, no ano de 2000, foi de 145.968 toneladas, sendo o Rio Grande do Sul o maior produtor, com 91.681 toneladas (Agrianual, 2003). Neste Estado, a produtividade é muito baixa (Raseira \& Nakasu, 1998), se comparada à média obtida no Estado de São Paulo, que é, pelo menos, duas vezes maior (Maia et al., 1996). Dentre os principais problemas que afetam o pessegueiro, os nematóides fitoparasitas podem causar o declínio do pomar, afetando a sua produtividade e, em casos extremos, levar a planta à morte. No Brasil, não existem estimativas de perdas na produtividade do pessegueiro em função do ataque de fitonematóides, mas, nos Estados Unidos, Anon (1971) estimou em $15 \%$. Desta forma, o emprego de porta-enxertos resistentes a nematóides pode diminuir os prejuízos causados por estes parasitas na cultura.

O nematóide das galhas (Meloidogyne spp.) é considerado um dos principais nematóides que atacam o pessegueiro nas mais variadas regiões produtoras do mundo. Plantas atacadas apresentam a formação de galhas nas raízes, a paralisação do crescimento e morte de pontas de raízes. As plantas podem apresentar depauperamento e declínio lento, deficiência nutricional, diminuição do tamanho das folhas e frutos, e redução da produtividade (Carneiro, 1998).

No Estado de São Paulo, o cv. Okinawa é o porta-enxerto mais utilizado (Barbosa et al., 1993), por apresentar, dentre outras características, resistência ou imunidade ao nematóide de galhas (Menten et al.,
1977; Scherb et al., 1994, Fachinello et al., 2000). A propagação desse material é feita por sementes, o que traz como conseqüência a variabilidade genética. Segundo Barbosa et al. (1993), esta prática pode fazer com que certa porcentagem dos descendentes se tornem suscetíveis aos nematóides do gênero Meloidogyne spp.

A variabilidade genética da cv. Okinawa, provocada pela propagação sexuada, e a utilização, no Rio Grande do Sul, de sementes de diversas cultivares e sem identidade genética conhecida, obtidas nas indústrias de conservas, acarretam diferentes reações dos porta-enxertos aos fitonematóides, dificultando as medidas de controle (Gomes, 2001). Face a esta realidade, pesquisas devem ser realizadas no sentido de identificar fontes de resistência em materiais com potencial para o Brasil e, a partir daí, propagá-los vegetativamente, mantendo a identidade da planta-matriz.

O umezeiro (Prunus mume Sieb. et Zucc.) é uma frutífera de folhas caducas da família Rosaceae e é típica de clima temperado. Alguns estudos foram realizados no Brasil, objetivando sua utilização como porta-enxerto para pessegueiros e nectarineiras. Os resultados iniciais foram bastante promissores, destacando-se a compatibilidade com Prunus persica, o aumento da massa, teor de sólidos solúveis e porcentagem de vermelho na película dos frutos, além da redução do vigor das plantas em até $50 \%$, quando comparadas às plantas enxertadas na cv. Okinawa (Campo Dall'Orto et al., 1992 e 1994, Nakamura et al., 1999).

$\mathrm{Na}$ Faculdade de Ciências Agrárias e Veterinárias - UNESP, Câmpus de Jaboticabal-SP, está sendo desenvolvido um amplo projeto de estudos sobre a utilização de clones de umezeiro, propagados por

\footnotetext{
${ }^{1}$ (Trabalho 162/2002). Recebido: 28/10/2002; Aceito para publicação: 26/03/2003. Apoio Financeiro: FAPESP. Parte da Tese de Doutorado do primeiro autor.

${ }^{2}$ Eng. Agr., M.Sc., Aluno do Curso de Pós-Graduação em Agronomia da Faculdade de Ciências Agrárias e Veterinárias, Câmpus de Jaboticabal. FCAV/UNESP, Departamento de Produção Vegetal, Via de Acesso Prof. Paulo Donato Castellane Km 05, CEP 14884-900, Jaboticabal-SP. Fone: (016) 3209-2668. E-mail: namayer@fcav.unesp.br.

${ }^{3}$ Eng. Agr., Dr., Prof. Titular do Departamento de Produção Vegetal da FCAV/UNESP, Câmpus de Jaboticabal-SP.

${ }^{4}$ Eng. Agr., Prof. Assistente Doutor do Departamento de Fitossanidade da FCAV/UNESP, Câmpus de Jaboticabal-SP.
} 
estacas herbáceas, como porta-enxerto para pessegueiro. Uma das etapas envolve estudos da reação destes clones a fitonematóides nocivos à persicultura. A literatura, embora escassa, sobre o assunto, classifica 0 umezeiro como altamente resistente a Meloidogyne spp. (Sherman \& Lyrene, 1983; Rossi et al., 2002), fato este que o coloca como uma alternativa para o problema, mas que merece maiores investigações.

Assim sendo, o objetivo do presente trabalho foi estudar a reação de clones de umezeiro e cultivares de pessegueiro a Meloidogyne javanica.

O experimento foi conduzido em casa de vegetação com cobertura plástica e laterais teladas, pertencente ao Departamento de Fitossanidade da FCAV/UNESP, Câmpus de Jaboticabal-SP. Foram utilizados vasos de cerâmica de 10 litros de capacidade, contendo substrato constituído de uma mistura de solo e areia $(1: 1, \mathrm{v} / \mathrm{v})$, previamente autoclavada a $121^{\circ} \mathrm{C}$ e $1 \mathrm{kgf.cm}{ }^{-2}$, por 2 horas.

Estacas herbáceas dos Clones 05; 10 e 15 de umezeiro e de pessegueiro cv. Okinawa, enraizadas em vermiculita, foram obtidas em câmara de nebulização intermitente e transplantadas para os vasos contendo o substrato esterilizado. As estacas de umezeiro foram obtidas de plantas-matrizes jovens mantidas em vasos sob ripado com $50 \%$ de luz natural. Utilizaram-se também plântulas das cultivares Aurora-1 e Dourado-1, cujas sementes foram obtidas de pomares localizados em TaiúvaSP e Jundiaí-SP (Estação Experimental de Jundiaí-IAC), respectivamente.

Foi utilizada como inóculo uma população de Meloidogyne javanica (Treub, 1885; Chitwood, 1949; Taylor \& Netscher, 1974; Hussey \& Barker, 1974; Eisenback et al., 1981), proveniente de um plantio comercial de tomateiro em Pelotas-RS. Sessenta dias após o transplante das mudas para os vasos, nove plantas de cada clone de umezeiro e das cultivares de pessegueiro foram inoculadas, individualmente, com uma suspensão de 3.000 ovos e juvenis de segundo estádio de $M$. javanica, aplicados com pipeta automática em quatro orifícios de $3 \mathrm{~cm}$ de profundidade, eqüidistantes entre si, no substrato ao redor da base das plantas. Cinco plantas de tomateiro cv. Santa Cruz Kada também foram inoculadas para a comprovação da eficiência do inóculo.

$\mathrm{O}$ experimento foi conduzido em delineamento inteiramente casualizado, com 6 tratamentos (Clones $05 ; 10$ e 15 de umezeiro e cultivares Okinawa, Aurora-1 e Dourado-1 de pessegueiro) e 9 repetições, sendo cada parcela constituída por uma planta. Transcorridos 100 dias após a inoculação, as plantas foram desenvasadas para avaliação das seguintes variáveis: massa de matéria fresca do sistema radicular, número de galhas por sistema radicular, número de ovos e juvenis por $10 \mathrm{~g}$ de raízes, número de ovos e juvenis por sistema radicular e fator de reprodução (FR), segundo Cook \& Evans (1987). Os dados foram transformados para $\log (\mathrm{x}+1)$ e submetidos à analise de variância pelo teste $\mathrm{F}$. As médias foram comparadas pelo teste de Tukey, ao nível de $5 \%$ de probabilidade. A definição dos graus de resistência dos diferentes genótipos foi baseada em Canto-Sáenz (1985).

De acordo com os resultados apresentados na Tabela 1 (médias originais), verificou-se que os três clones de umezeiro não apresentaram formações de galhas nas raízes. Entretanto, após a extração e contagem, foram encontradas médias entre 9,4 e 17,5 ovos e juvenis por sistema radicular, não diferindo estatisticamente entre si. Rossi et al. (2002) também não verificaram formação de galhas ou massa de ovos nas raízes de umezeiro provocadas por M. javanica, mas foi encontrada uma média de 6,3 nematóides por sistema radicular, o que pode indicar a existência de um mecanismo de resistência que envolve aspectos fisiológicos póspenetração de juvenis de segundo estádio, conforme mencionado por Scherb et al. (1994) para 'Okinawa' e 'R-15-2', em relação a M. incognita. Entretanto, em função de os valores do fator de reprodução (FR) serem muito inferiores a 1 , os clones foram considerados resistentes a $M$. javanica (Canto-Sáenz, 1985), o que concorda com relatos de Sherman \& Lyrene (1983) e Rossi et al. (2002) sobre a resistência do umezeiro a esta espécie de fitonematóide, sendo esta mais uma vantagem do uso deste material como porta-enxerto na persicultura moderna e intensiva. As raízes dos tomateiros foram severamente atacadas pelo nematóide, apresentando fator de reprodução igual a 1546, confirmando a viabilidade do inóculo utilizado.

TABELA 1- Reação de clones de umezeiro e cultivares de pessegueiro a uma população inicial de 3000 ovos e juvenis de Meloidogyne javanica, aos 100 dias após a inoculação. Jaboticabal-SP, junho de 2002.

\begin{tabular}{cccccc}
\hline Genótipo & $\begin{array}{c}\mathrm{n}^{\circ} \\
\text { galhas/sist. } \\
\text { Radicular }\end{array}$ & $\begin{array}{c}\mathrm{n}^{\circ} \text { de ovos e } \\
\text { juvenis/10 } \\
\text { raízes }\end{array}$ & $\begin{array}{c}\mathrm{n}^{\circ} \text { de ovos e } \\
\text { juvenis/sist. } \\
\text { Radicular }\end{array}$ & FR & Reação \\
\hline Clone 05 & $0 \mathrm{~b}$ & $5,6 \mathrm{~b}$ & $17,5 \mathrm{~b}$ & 0,0058 & $\mathrm{R}$ \\
Clone 10 & $0 \mathrm{~b}$ & $2,8 \mathrm{~b}$ & $10,3 \mathrm{~b}$ & 0,0034 & $\mathrm{R}$ \\
Clone 15 & $0 \mathrm{~b}$ & $2,8 \mathrm{~b}$ & $9,4 \mathrm{~b}$ & 0,0031 & $\mathrm{R}$ \\
Okinawa & $0 \mathrm{~b}$ & $0 \mathrm{~b}$ & $0 \mathrm{~b}$ & 0 & $\mathrm{R}$ \\
Aurora-1 & $9,6 \mathrm{a}$ & $36,1 \mathrm{a}$ & $200,1 \mathrm{a}$ & 0,0667 & $\mathrm{R}$ \\
Dourado-1 & $7,2 \mathrm{a}$ & $0 \mathrm{~b}$ & $0 \mathrm{~b}$ & 0 & $\mathrm{R}$ \\
\hline $\mathrm{F}$ & $32,73^{* *}$ & $8,75^{* *}$ & $9,94 * *$ & - & - \\
\hline
\end{tabular}

** significativo ao nível de $1 \%$ de probabilidade. Médias seguidas por letras distintas, na mesma coluna, diferem entre si pelo teste de Tukey, ao nível de $5 \%$ de probabilidade. $\mathrm{R}=$ resistente. Observação: Fator de Reprodução do tomateiro cv. Santa Cruz $=1546$.

Não foram encontrados galhas, ovos ou juvenis nas raízes de pessegueiro cv. Okinawa, confirmando a resistência deste porta-enxerto, previamente observada por Menten et al. (1977), Scherb et al. (1994) e Fachinello et al. (2000).

Nas cultivares-copa de pessegueiro Aurora-1 e Dourado-1, detectou-se um pequeno número de galhas de reduzido tamanho, que não diferiram estatisticamente entre si, mas diferiram de todos os outros genótipos testados. Entre as cultivares de pessegueiro testadas, um reduzido número de ovos e juvenis por $10 \mathrm{~g}$ de raízes e por sistema radicular foi encontrado somente nas raízes da cultivar Aurora-1 (Tabela 1), diferindo estatisticamente das demais. Entretanto, considerando-se os valores do fator de reprodução, todas as cultivares de pessegueiro testadas mostraram-se resistentes ao nematóide, conforme a terminologia proposta por Canto-Sáenz (1985). A cultivar Dourado-1 tem como origem o cruzamento 'Tutu' $\mathrm{x}$ 'Maravilha', sendo que este último tem como um dos progenitores a cv. Okinawa (Ojima et al., 1985), o que pode explicar a reação de resistência da cv. Dourado-1 ao nematóide.

Nas condições experimentais utilizadas, os clones $05 ; 10$ e 15 de umezeiro, assim como as cultivares de pessegueiro Okinawa, Aurora1 e Dourado-1, mostraram-se resistentes a Meloidogyne javanica. A resistência dos clones de umezeiro a Meloidogyne javanica, aliada às demais qualidades agronômicas como porta-enxerto, pode contribuir para o aumento da produtividade da cultura do pessegueiro.

\section{REFERÊNCIAS BIBLIOGRÁFICAS}

AGRIANUAL 2003: anuário da agricultura brasileira. São Paulo: FNP Consultoria \& Comércio, 2003. p.446-450.

ANON, A. Estimated crop losses due to plant parasitic nematodes in the United States. Nematology, v.1, p.7, 1971.

BARBOSA, W.; CAMPO DALL'ORTO, F.A.; OJIMA, M.; MARTINS, F.P. Produção e manejo de sementes do pessegueiro porta-enxerto Okinawa. O Agronômico, Campinas, v.45, n.2/3, p.10-16, 1993.

CAMPO DALL'ORTO, F.A.; OJIMA, M.; BARBOSA, W.; MARTINS, F.P. O nanismo do pessegueiro induzido pela enxertia no damasqueiro-japonês. Pesquisa Agropecuária Brasileira, Brasília, v.27, n.3, p.517-521, 1992

CAMPO DALL'ORTO, F.A.; BARBOSA, W.; OJIMA, M.; MARTINS, F.P.; FOBÉ, L.A. Comportamento de pessegueiros IAC enxertados no damasqueiro-japonês e no pessegueiro 'Okinawa'. In: CONGRESSO BRASILEIRO DE FRUTICULTURA, 13., 1994, Salvador. Anais... Cruz das Almas: SBF, 1994. v.3, p.879-880.

CANTO-SÁENZ, M. The nature of resistance to Meloidogyne incognita (Kofoid \& White, 1919) Chitwood, 1949. In: SASSER , J.N.; 
CARTER, C.C. An advanced treatise on Meloidogyne; biology and control. Raleigh: North Carolina State University Graphics, 1985. p.225-231.

CARNEIRO, R.M.D.G. Nematóides: ocorrência e danos. In: MEDEIROS, C.A.B.; RASEIRA, M. do C.B. A cultura do pessegueiro. Pelotas: Embrapa-CPACT, 1998.p.265-279.

COOK, R.; EVANS, K. Resistance and tolerance. In: BROWN, R.H.; KERRY, B.R. (Ed.) Principles and practice of nematode control in crops. New York: Academic Press, 1987. p.179-231.

EISENBACK, J.D.; HIRSCHMANN, H.; SASSER, J.N.; TRIANTAPHYLLOU,A.C. A guide to the four most common species of root-knot nematodes (Meloidogyne species) with a pictorial key. Raleigh: The Departments of Plant Pathology and Genetics of North Carolina State University and United States Agency for International Development, 1981.48p.

FACHINELLO, J.C.; SILVA, C.A.P.; SPERANDIO, C.; RODRIGUES,A.C.; STRELOW, E.Z. Resistência de porta-enxertos para pessegueiro e ameixeira aos nematóides causadores de galhas (Meloidogyne spp.). Ciência Rural, Santa Maria, v.30, n.1, p.69-72, 2000.

GOMES, C.B. Problemas causados por nematóides em fruteiras de clima temperado. In: CONGRESSO BRASILEIRO DENEMATOLOGIA, 23., 2001, Marília. Anais... Garça: Sociedade Brasileira de Nematologia, 2001.p.45-51.

HUSSEY, R.S.; BARKER, K.R. A comparison of methods of collecting inocula of Meloidogyne spp. including a new technique. Plant Disease Reporter, St. Paul, v.57, p.1025-1028, 1974.

MAIA, M.L.; AMARO, A.A.; GONÇALVES, J.S.; SOUZA, S.A.M. Produção e mercado de pêra e pêssego no Brasil. Informações Econômicas, São Paulo, v.26, n.2, p.33-48, 1996.

MENTEN, J.O.M.; LORDELLO, L.G.E.; CAMPO DALL'ORTO, F.A.; OJIMA, M.; RIGITANO, O. Resistência varietal de pessegueiro
(Prunus persica Batsch) aos nematóides Meloidogyne incognita e M. arenaria. In: REUNIÃO DE NEMATOLOGIA, 2., 1977, Piracicaba. Trabalhos apresentados... Piracicaba: Franciscana, 1977. v.2, n.2, p.165-173.

NAKAMURA, C.H.; SCARPARE FILHO, J.A.; KLUGE, R.A. Avaliação preliminar do umezeiro como porta-enxerto para pessegueiro e nectarineira. Revista Brasileira de Fruticultura, Jaboticabal, v.21, n.2,p.116-118, 1999.

OJIMA, M.; CAMPO DALL'ORTO, F.A.; BARBOSA, W.; TOMBOLATO, A.F.C.; RIGITANO, O.; SCARANARI, H.J.; MARTINS, F.P.; SANTOS, R.R. dos. 'Dourado-1' e 'Dourado-2': novas cultivares de pêssego-amarelo para mesa. Bragantia, Campinas, v.44, n.1, p.451-455, 1985.

RASEIRA, M. do C.B.; NAKASU, B.H. Situação e perspectivas do cultivo de fruteiras de clima temperado no Rio Grande do Sul - Frutas de caroço. In: REUNIÃO TÉCNICA DE FRUTICULTURA, 5., 1998, Veranópolis. Anais... Porto Alegre: Fepagro, 1998. p.21-22.

ROSSI, C.E; FERRAZ, L.C.C.B.; MONTALDI, P.T. Resistência de frutíferas de clima subtropical e temperado a Meloidogyne incognita raça 2 e M. javanica. Arquivos do Instituto Biológico, São Paulo, v.69, n.2, p.43-49, abr./jun., 2002.

SCHERB, C.T.; CAMPOS, V.P.; CHALFUN, N.N.J. Penetração e reprodução de Meloidogyne incognita em pessegueiro das variedades Okinawa e R-15-2. Revista Brasileira de Fruticultura, Cruz das Almas, v.16. n.1, p.134-138, 1994.

SHERMAN, W.B.; LYRENE, P.M. Improvement of peach rootstock resistant to root-knot nematodes. Proceedings of the Florida State for Horticultural Society, v.96, p.207-208, 1983.

TAYLOR, A. L.; NETSCHER, C. An improved technique for preparing perineal patterns of Meloidogyne spp. Nematologica, Leiden, v.20, p.268-269, 1974. 